\title{
17. Novel Coulombic Complexes of Amphiphilic Polyelectrolytes and Double-chain Surfactants
}

\author{
By Yotaro Morishima, Michiko SEKI, Shigeki Nomura, \\ and Mikiharu KAMACHI \\ Department of Macromolecular Science, Faculty of Science, Osaka University, \\ Toyonaka, Osaka 560 \\ (Communicated by Seizo OKamura, M. J. A., April 12, 1993)
}

\begin{abstract}
Amphiphilic polysulfonates containing 1-adamantyl (Ad) or 1naphthylmethyl $(\mathrm{Np})$ groups were treated with didodecyldimethylammonium bromide (DDAB) (double-chain cationic surfactant) to yield Coulombic complexes which were soluble in common organic solvents. ${ }^{1} \mathrm{H}-\mathrm{NMR}$ spectra, $2 \mathrm{D}-\mathrm{NOESY}$, and NMR relaxation times for the complex of the Np-containing polymer in organic solution revealed that the motions of the Np residues were highly restricted, while those of the DDAB residues were much less restricted. These findings imply that the micelle structure of the parent amphiphilic polysulfonates in aqueous solution remained rather intact in the complexes even though they were dissolved in organic solvents. Covalently attached spin probes indicated that the complex was in a particularly compact form in benzene. Fluorescence emitted by pyrene incorporated in the complexes supported these findings.
\end{abstract}

Key words: Amphiphilic polyelectrolyte; polymer micelle; coulombic complexes; NMR relaxation time; ESR spin probe.

Introduction. An amphiphilic polyelectrolyte having a sufficiently high mole fraction of bulky hydrophobic pendant groups forms a unimolecular micelle in dilute aqueous solution due to intramolecular self-organization of the hydrophobic pendant groups. When a small mole fraction of hydrophobic chromophores are covalently incorporated into the amphiphilic polyelectrolyte, they would be encapsulated in the hydrophobic domain of the micelle, leading to the "compartmentalization" of the chromophores. ${ }^{1-4)}$ We have reported that the compartmentalized chromophores show unique behavior in fluorescence and phosphorescence, ${ }^{5)}$ in energy transfer and migration, ${ }^{6)}$ in photoinduced electron transfer, ${ }^{1-3)}$ and in photoisomerization ${ }^{7)}$ in aqueous solution. From these studies we consider that the compartmentalization of chromophores is a promising strategy for designing useful photo-systems such as photon-harvesting, photoinduced charge separation, and photochromic systems. Our goal is to develop novel photochemical systems based on the compartmentalization of chromophores, which are soluble in organic solvents and hence in polymer solids. ${ }^{8)}$

As an approach to this goal, we prepared Coulombic complexes of the amphiphilic polyelectrolyte micelle with double-chain surfactants. These complexes were soluble in common organic solvents. However, an important question was whether the hydrophobic domain structure in the micelle remains intact in the complexes when they were dissolved in organic solvents. To answer this question, we investigated the dynamic properties and structure of the complexes by dynamic light scattering (DLS), GPC, ${ }^{1} \mathrm{H}-\mathrm{NMR}$ relaxation, 2D-NOESY, ESR spin probe, and fluorescence techniques in various organic solvents.

Experimental section. Amphiphilic polyelectrolytes were prepared by free-radical terpolymerization of 0.50 mole fraction of sodium 2-(acrylamido)-2-methylpropane- 
sulfonate (AMPS), comparable mole fractions of methacrylamides bearing bulky hydrophobic groups, and small mole fractions of methacrylamides bearing spectroscopic probes. As bulky hydrophobic substituents, 1-adamantyl (Ad) and 1-naphthylmethyl (Np) groups were employed. As probes, 1-pyrenyl (Py) and 2, 2, 6, 6-tetramethylpiperidine-N-oxide (NO) groups were employed. The terpolymerizations were performed in $\mathrm{N}, \mathrm{N}$ dimethylformamide (DMF) solution at $60^{\circ} \mathrm{C}$ in the presence of $2,2^{\prime}$-azobis (isobutyronitrile). The compositions of the terpolymers were determined by elemental analysis and absorption spectroscopy, finding that they were virtually the same as the monomer feed compositions. The polymers were purified by dialysis against pure water for several days and recovered by freeze-drying.

The Coulombic complexes (Scheme I) were prepared as follows. A 0.3-m $M$ aqueous solution of didodecyldimethylammonium bromide (DDAB) was added to a $0.3-w t \%$ aqueous solution of the terpolymer to yield fine particles, which were collected by centrifugation and suspended in pure water. The complexes in the water suspension were purified by dialysis and recovered by freeze-drying.

Particle size distributions were estimated with an Otsuka Electronics DLS-700 spectrophotometer at $40^{\circ} \mathrm{C}$. Two-dimensional nuclear Overhauser enhancement spectra (2D-NOESY) were obtained with a JEOL EX-270 spectrometer at $25^{\circ} \mathrm{C}$. Three contour levels were plotted in a sequence of $\times(1 / 4), \times(1 / 2)$, and 1 as the maximum threshold values. Spin-lattice relaxation times in ${ }^{1} \mathrm{H}-\mathrm{NMR}\left(T_{1}\right)$ were determined by the inversion-recovery technique with $180^{\circ}-\tau-90^{\circ}$ pulse sequences. ${ }^{9)-11)}$ Spin-spin relaxation times $\left(T_{2}\right)$ were determined by the CPMG method. ${ }^{12)}$ ESR spectra were recorded with a JEOL JES-RE2X spectrometer. Fluorescence spectra were measured on a Hitachi 850 spectrometer.

Results and discussion. In scheme I are shown the chemical formulas of the Coulombic complexes prepared in the present study. The complexes and their parent terpolymers are abbreviated as poly $(\mathrm{A} / \mathrm{R} / \mathrm{P}) / \mathrm{DDAB}$ and poly $(\mathrm{A} / \mathrm{R} / \mathrm{P})$, respectively, where $\mathrm{A}, \mathrm{R}$, and $\mathrm{P}$ represent the AMPS, hydrophobic, and probe units, respectively, as indicated in Scheme I. In dilute aqueous solution of the amphiphilic polyelectrolytes, the formation of the micellar structure is completely due to the intramolecular self-organization of the hydrophobic groups. ${ }^{13)}$ In a $0.32 \mathrm{wt} \%$ aqueous solution of poly (A/Ad/Py), for example, the

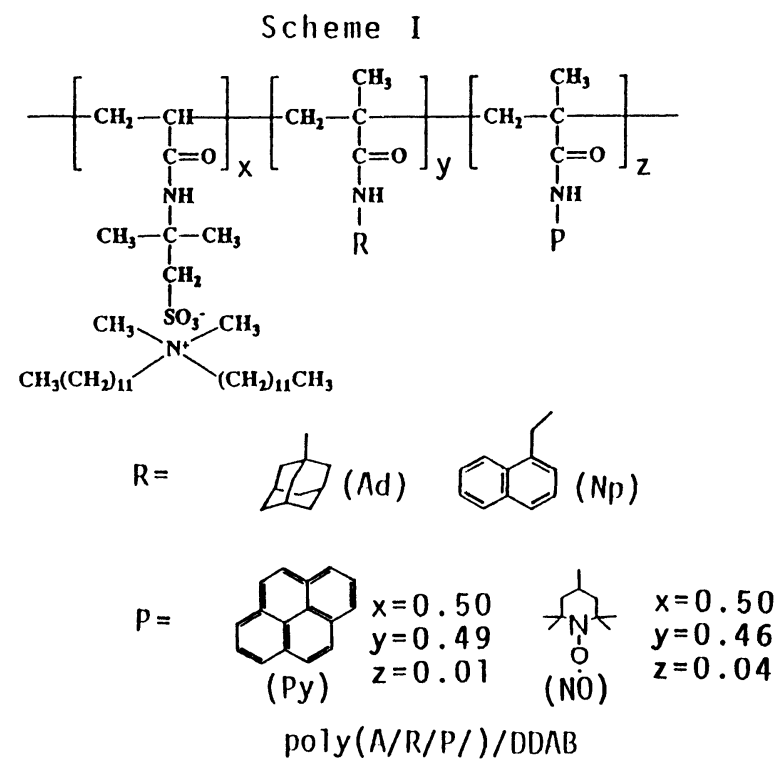


size of the unimolecular micelle was distributed in the range from 13 to $21 \mathrm{~nm}$ in diameter (Fig. 1a). When an aqueous solution of DDAB was added to the poly (A/Ad/Py) solution, the solution became turbid due to phase separation of the resulting complexes whose particle size was estimated to range from 60 to $250 \mathrm{~nm}$ as shown in Fig. 1b.

Fig. 2 compares 2D-NOESY spectra of poly $(\mathrm{A} / \mathrm{Np} / \mathrm{Py})$ in $\mathrm{D}_{2} \mathrm{O}$ and poly $(\mathrm{A} / \mathrm{Np} / \mathrm{Py}) /$ DDAB in benzene- $d_{6}$. The cross peaks between $\mathrm{Np}$ and aliphatic protons observed for both poly $(\mathrm{A} / \mathrm{Np} / \mathrm{Py})$ and poly $(\mathrm{A} / \mathrm{Np} / \mathrm{Py}) / \mathrm{DDAB}$ indicate that the conformation of the complex in benzene solution is as compact as that of the parent terpolymer in aqueous solution. To clarify the dynamic properties of the complex, $T_{1}$ and $T_{2}$ of ${ }^{1} \mathrm{H}-\mathrm{NMR}$ were estimated. Fig. 3 shows stack plots for the measurements of the ${ }^{1} \mathrm{H}-\mathrm{NMR}$ relaxation times for poly $(\mathrm{A} / \mathrm{Np} / \mathrm{Py}) / \mathrm{DDAB}$ in benzene- $d_{6}$ at room temperature. The values of $T_{1}$ and $T_{2}$ calculated from the data shown in Fig. 3 are listed in Table I. $T_{2}$ for the Np protons in poly $(\mathrm{A} / \mathrm{Np} / \mathrm{Py})$ in $\mathrm{D}_{2} \mathrm{O}$ was very small, being 1 order of magnitude smaller than that in DMF- $d_{7}$. The $T_{2}$ values for the $\mathrm{Np}$ protons of poly $(\mathrm{A} / \mathrm{Np} / \mathrm{Py}) / \mathrm{DDAB}$ were much smaller than those for the DDAB protons in the complex. Importantly, both $T_{1}$ and $T_{2}$ for the complex in benzene- $d_{6}$ were smaller than those in DMF- $d_{7}$, indicating that the conformation was more compact in benzene than in DMF.

Fig. 4 shows ESR spectra of the parent terpolymer poly $(\mathrm{A} / \mathrm{Np} / \mathrm{NO})$ in aqueous
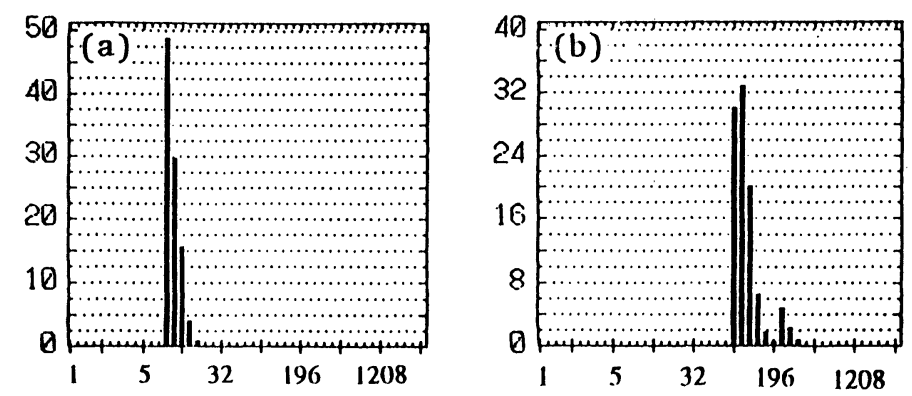

Diameter / nm

Fig. 1. Particle size distributions of poly $(\mathrm{A} / \mathrm{Ad} / \mathrm{Py})(\mathrm{a})$ and poly $(\mathrm{A} / \mathrm{Ad} /$ $\mathrm{Py}) / \mathrm{DDAB}(\mathrm{b})$ in water.

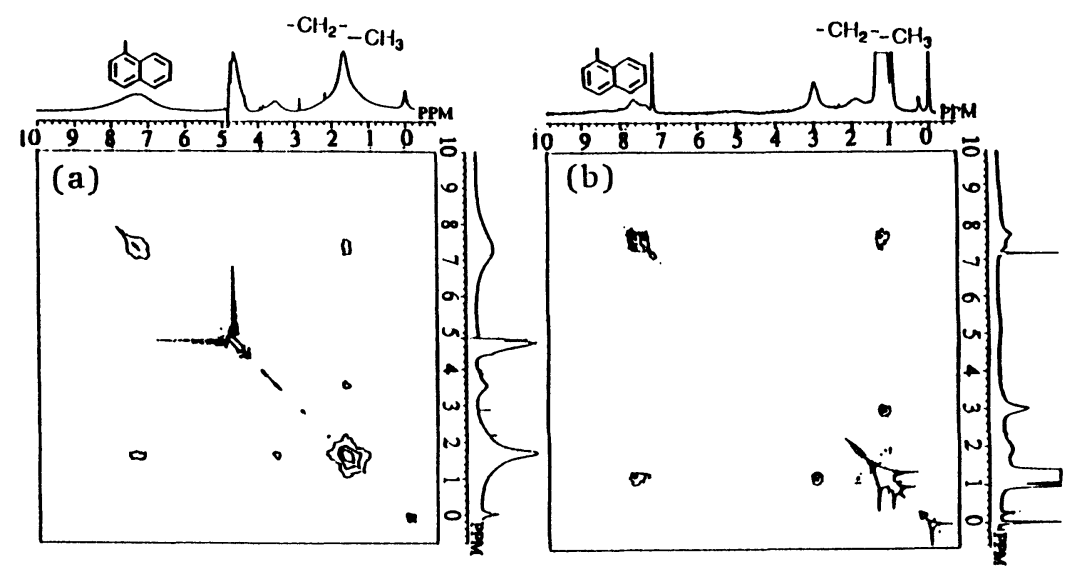

Fig. 2. 2D-NOESY contour plots for poly (A/Np/Py) in $\mathrm{D}_{2} \mathrm{O}$ (a) and poly $(\mathrm{A} / \mathrm{Np} / \mathrm{Py}) / \mathrm{DDAB}$ in benzene- $d_{6}(\mathrm{~b})$. 
[Vol. 69(B),

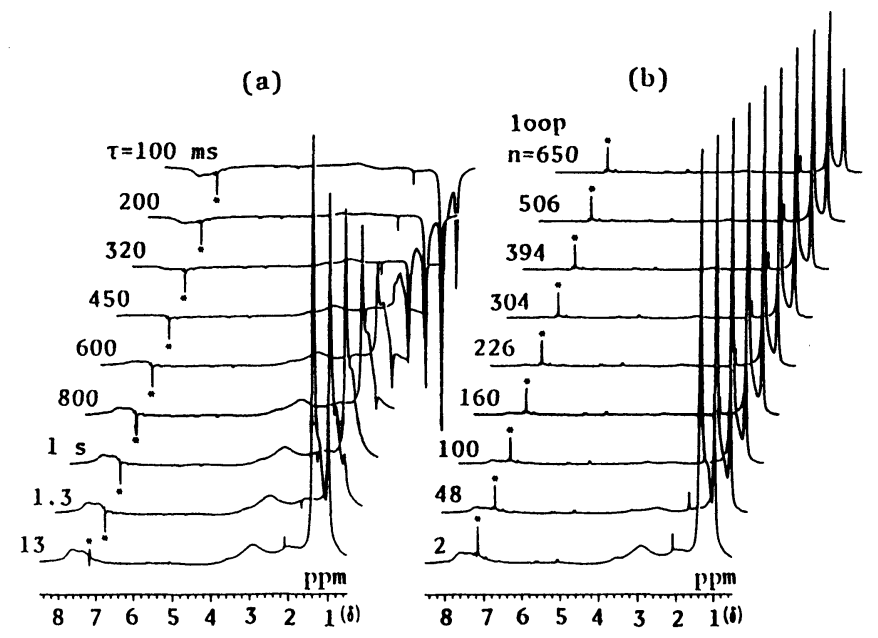

Fig. 3. Stack plots for poly (A/Np/Py)/DDAB, from which $T_{1}$ (a) and $T_{2}$ (b) were determined.

Table I. ${ }^{1} \mathrm{H}-\mathrm{NMR}$ relaxation times for the $\mathrm{Np}$ and $\mathrm{DDAB}$ methylene protons

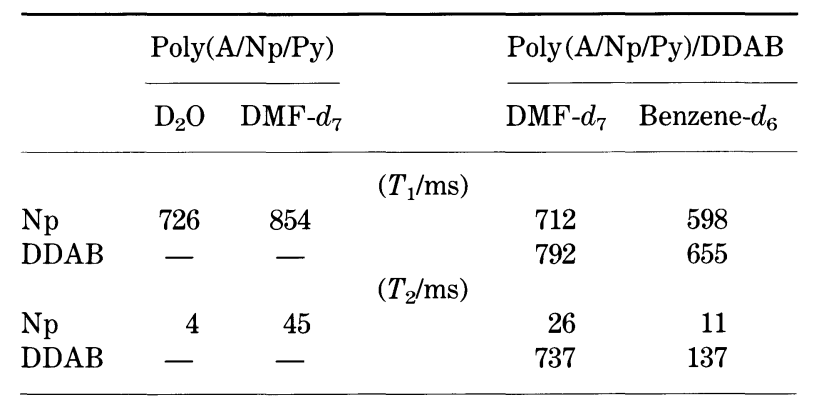

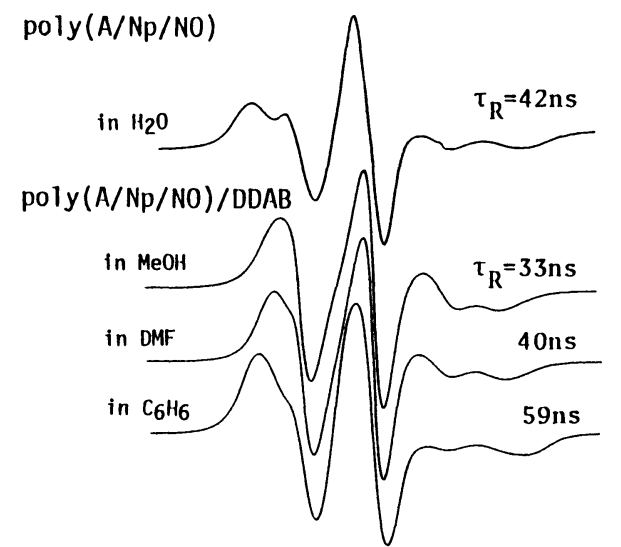

Fig. 4. ESR signals of poly $(\mathrm{A} / \mathrm{Np} / \mathrm{NO})$ in water and poly $(\mathrm{A} / \mathrm{Np} / \mathrm{NO}) /$ $\mathrm{DDAB}$ in various organic solvents. 
solution and the complex poly $(\mathrm{A} / \mathrm{Np} / \mathrm{NO}) / \mathrm{DDAB}$ in various solvents. The rotational correlation times $\left(\tau_{\mathrm{R}}\right)$ estimated by simulation are indicated in the figure. $\tau_{\mathrm{R}}$ of the NO probes in the complex depended on the solvent, indicating that the motions of the NO probes were restricted in the solvents in the order of $\mathrm{MeOH}<\mathrm{DMF}<$ benzene. It should be noted that the motions of the NO probes in the complex in benzene were even slower than those of the parent terpolymer in water. These results indicate that, in benzene, the complexes retain the micellar structure of the parent polymers. A conceptual illustration of the complex is presented in Fig. 5.

Fluorescence emitted from the pyrene probes provides information about the nature of their local environments, because the ratio of the third to first vibrational fine structure, $I_{3} / I_{1}$, of the pyrene fluorescence is dependent on local environmental polarity, the $I_{3} / I_{1}$ ratio being higher in less polar media. ${ }^{14)}$ Fig. 6 compares fluorescence spectra emitted by the Py probes in poly (A/Ad/Py)/DDAB in benzene and in DMF. The values of $I_{3} / I_{1}$ for poly $(\mathrm{A} / \mathrm{Ad} / \mathrm{Py}) / \mathrm{DDAB}$ in benzene and in $\mathrm{DMF}$ are very similar although the solvent polarities are different, indicating that the Py probes in the complex experience micropolarities which are different from the solvent polarities and that they are confined to microenvironments which are separated from the solvent phase. In poly $(\mathrm{A} / \mathrm{Ad} / \mathrm{Py}) / \mathrm{DDAB}$, the Py probes are surrounded by the Ad groups and also by ion pair groups. Therefore, the Py probes may not be exposed to the solvent phase but experience a polar microenvironment owing to the surrounding ion pairs in the complex even though they are in benzene solution.

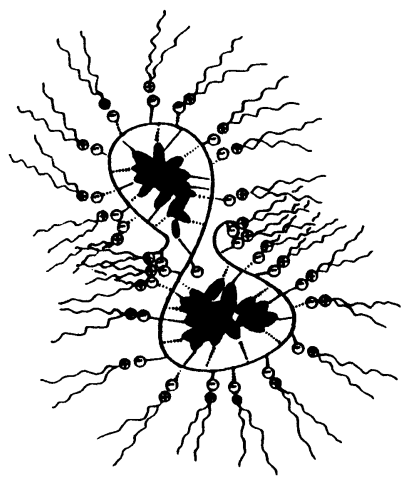

Fig. 5. Conceptual illustration of a local structure of the complex.

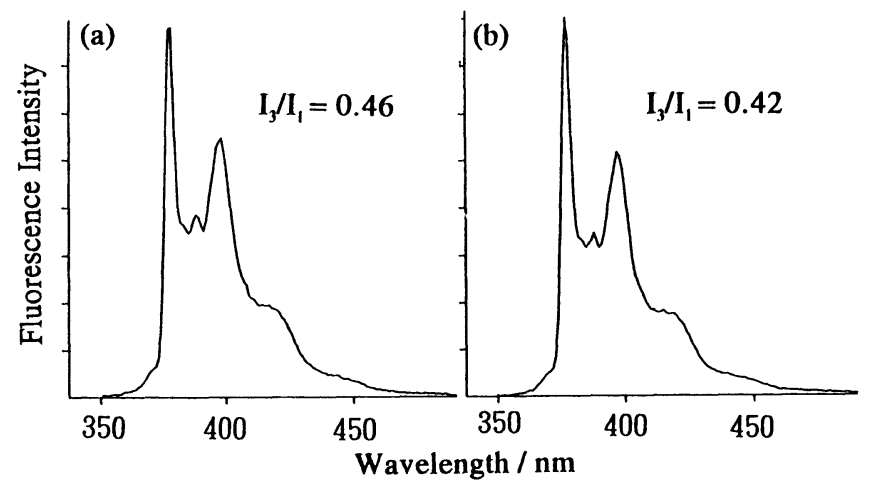

Fig. 6. Fluorescence spectra of poly $(\mathrm{A} / \mathrm{Ad} / \mathrm{Py}) / \mathrm{DDAB}$ in benzene (a) and in DMF (b). The $I_{3} / I_{1}$ ratios are indicated in the figure. 
[Vol. 69(B),

As will be reported in detail in the subsequent paper, ${ }^{15)}$ the amphiphilic terpolymer poly $(\mathrm{A} / \mathrm{Ad} / \mathrm{Py})$ was treated with viologen dications together with $\mathrm{DDAB}$ in aqueous solution to obtain a functionalized complex in which the Py moiety and viologen act as a photodonor and an electron acceptor, respectively. This complex exhibited a long-lived charge separated state in the photoinduced electron transfer in benzene solution, which is characteristic of the compartmentalization effect. Therefore this is an indication that, in benzene, the complex retains the micellar structure of the parent polymer as conceptually illustrated in Fig. 5 .

\section{References}

1) Y. Morishima et al.: Macromolecules, 20, 1707 (1987).

2) Y. Morishima et al.: J. Phys. Chem., 93, 1643 (1989).

3) Y. Morishima et al.: ibid., 95, 6027 (1991).

4) Y. Morishima: Adv. Polym. Sci., 104, 51 (1992).

5) H. Aota, Y. Morishima, and K. Kamachi: Photochem. Photobiol. (in press).

6) Y. Morishima et al.: Macromolecules, 96, 1990 (1992).

7) Y. Morishima et al.: ibid., 25, 4406 (1992).

8) Y. Morishima et al.: J. Polym. Sci., Polym. Chem. Ed., 30, 2099 (1992).

9) K. Erdmann and A. Gutsze: Colloid Polym. Sci., 256, 667 (1987).

10) P. Raby et al.: J. Polym. Sci., Polym. Phys. Ed., 29, 451 (1991).

11) M. G. Brereton et al.: Macromolecules, 24, 2068 (1991).

12) S. Meiboom and D. Gill: Rev. Sci. Instrum., 29, 688 (1958).

13) S. Nomura, Y. Morishima, and M. Kamachi: Macromolecules (to be submitted).

14) K. Kalyanasundaram and J. K. Thomas: J. Am. Chem. Soc., 99, 2039 (1977).

15) M. Seki, Y. Morishima, and M. Kamachi: Macromolecules (to be submitted). 